\title{
A Case Study of the Application of COTS Components in a Molecular Dynamics Software
}

\author{
N. Gnanasankaran, S. Natarajan, K. Alagarsamy, and K. Iyakutti
}

\begin{abstract}
Commercial Off-The-Shelf (COTS) components are widely used in many software industries and also in scientific computing. This paper first considers the definition of the term COTS and then tries to explore how it typically manifests itself in a software used for Molecular dynamical calculations. Some details regarding a popular software used for such calculations, viz., GROMACS are furnished. Since this is a freely downloadable program and a non-commercial one, it is better to consider this as an example of Scientific Off -TheShelf (SOTS) components, a newly coined acronym.
\end{abstract}

Index Terms-COTS, GROMACS software, molecular dynamics, SOTS.

\section{INTRODUCTION}

COTS components are defined as "components which are bought from third party vendors and integrated into the system". However, a more detailed and expanded view of COTS components should be taken. A COTS component could be as small as a routine that computes the square root of a number or as large as a credit card validation software. The important thing is that a COTS component already exists and was created by people outside the software development organization that will actually use it.

A commercial off-the-shelf component can therefore be defined as, "any software component that already exists, that was created by people outside the organization that will be using it, and that was purchased from a third party vendor" [1]. [1]:

The above definition leads to the following conclusions

1) The source code can never be made available to the component user, unlike an in-house component. A COTS component is like a black box. The user can view the component only through its interfaces and outputs.

2) There are possibilities that a complete description of the component's behavior may not be given by the vendor to the user. This may result in problems during component integration.

3) Maintenance can become an issue because the vendor

Manuscript received November 12, 2012; revised March 27, 2013.

N. Gnanasankaran is with the Department of Master of Computer Applications, KLN College of Information Technology, Pottapalayam, Tamil Nadu, India (email: sankarn.iisc@ gmail.com).

S. Natarajan is with the School of Physics, Madurai Kamaraj University, Madurai, Tamil Nadu, India.

K. Alagarsamy is with the Computer Centre, Madurai Kamaraj University, Madurai, Tamil Nadu, India.

K. Iyakutti is with the Department of Physics, Noorul Islam University, Kumaracoil, Tamil Nadu, India. may not correct defects or add enhancements according to the component's specification.

4) Sometimes the vendors can provide updated components that do not integrate with the earlier version of the application.

Component-based development offers several focus of reuse. Some common sources from where one can acquire components are:

1) External component libraries

2) Commercial off-the-shelf (COTS) components

3) Open source software (OSS) components

4) In-house component infrastructure

5) Mining components from legacy systems

Almost without exception, every software-related endeavour will utilize a significant percentage of COTS software components. The application of COTS components in crystallographic softwares was evaluated by us earlier [2], [3]. In another contribution, the case of the mathematical software viz., SCILAB was taken up as a case study [4]. In this paper, the case of a molecular dynamics software is taken up for detailed study. Since the program package to be discussed in the following sections is freely downloadable from websites and a non-commercial one, it is better to consider this as an example of Scientific Off-The-Shelf (SOTS) components, a newly coined acronym.

\section{ENERGY MINIMIZATION}

In computational chemistry or physics, energy minimization (also called energy optimization or geometry optimization) methods are used to compute the equilibrium configuration of molecules and solids. Starting from a non-equilibrium molecular geometry, energy minimization employs the mathematical procedure of optimization to move atoms so as to reduce the net forces on the atoms until they become negligible. A well established algorithm of energy minimization can be an efficient tool for molecular structure optimization.

The algorithms of gradient are the most popular methods for energy minimization. The basic idea of gradient methods is to move atoms by the total net forces acting on them. The force on atoms is calculated as the negative gradient of total potential energy of the system. An analytical formula of the gradient potential energy is preferentially required by the gradient methods. If not, one needs to calculate numerically the derivatives of the energy function from the potential. Fig. 1 shows the schematics of the algorithm used [5].

Molecular modeling methods are now routinely used to investigate the structure, dynamics, surface properties and thermodynamics of inorganic, biological and polymeric 
systems. The types of biological activities that have been investigated using molecular modeling include protein folding, enzyme catalysis, protein stability, conformational changes associated with bio-molecular function and molecular recognition of proteins, DNA and membrane complexes.

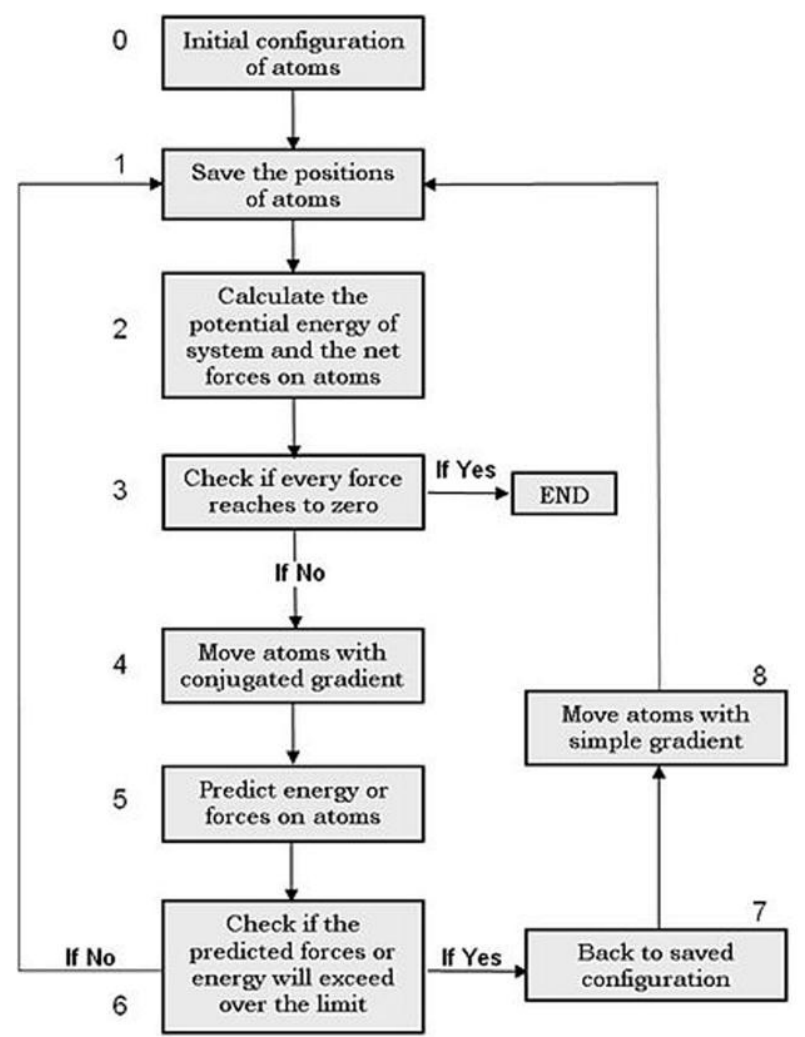

Fig. 1. Schematics of a computational self consistent energy minimization procedure.

\section{SOFTWARES FOR MOleCUlar MODELING}

There are numerous software packages for the above calculations in molecular dynamics. Some of the popular packages are AMBER, Auto Dock, CHARMM, VASP, GAUSSIAN, GROMACS, Materials Studio, Sirius, etc. In the following sections, some of the features of the software: GROMACS [5] are discussed.

\section{A. The GROMACS Software}

GROMACS (Groningen Machine for Chemical Simulations) is a molecular dynamics simulation package originally developed in the University of Groningen. It is written in $\mathrm{C}, \mathrm{C}++$ and Assembly languages and may be used in Solaris. Linux, MacOS X, Windows and Unix operating systems.

1) GROMACS is an engine to perform molecular dynamics simulations and energy minimization.

2) A high performance Molecular Dynamics Program.

3) It is reasonably well optimized for low memory usage.

4) The highly optimized code makes GROMACS the fastest program for molecular simulations.

5) The support of different force fields and the open source (GPL) character make GROMACS very flexible.

6) It is a high performance research tool which can be run on almost every platform.

7) This program is a free software; it can be redistributed.

\section{COMPUTATIONS INVOLVED}

Several steps of energy minimization may be necessary, which consist of the following cycles of computations.

Generating topologies and coordinates, running a simulation, converting pdb files to topology and coordinate files, generating a primitive topology from coordinates, editing the box and writing subgroups, solvating a system, generating mono atomic ions on energetically favorable positions, multiplying a conformation in 'random' orientations, generating position restraints or distance restraints for index groups, protonating structures , making a run input file, making a run input file for restarting a crashed run and performing a simulation.

From the details given in the above paragraph, it is easy to understand that one has to go through several computations, step by step to arrive at the minimum energy structure of the molecule, using the data available. Suitable computer programs have been written and are available for the different steps of the calculations, stated above. The modern software package such as GROMACS incorporates all the above programs in a unique way and helps the scientists in arriving at the minimum energy configuration of the molecules. No one knows the details of the programs used in the above packages and they remain black boxes, but help the scientists!

\section{CONClusions}

One could easily visualize the usage of (Non) Commercial Off-The-Shelf (COTS) technology in the above example. The modern packages are very versatile in their functioning and the quality is not compromised in utilizing the above software package. Hence, the above example could be considered as a positive usage of COTS components, in designing a software package for the usage of a group of scientists. Since the program package discussed above is freely downloadable from websites and a non-commercial one, it will be more appropriate to consider this as an example of Scientific Off-The-Shelf (SOTS) components, an acronym coined by us.

\section{ACKNOWLEDGEMENTS}

F. A. thanks the Management and the Principal of K.L.N. College of Information Technology, for the encouragements

\section{REFERENCES}

[1] S. Kalaimagal and R. Srinivasan, "A TRUSTAD component nomenclature," Journal of Object Technology, vol. 7, pp. 159-173, May-June, 2008.

[2] N. Gnanasankaran, S. Natarajan, K. Alagarsamy, and K. Iyakutti, "Application of COTS components in crystallographic softwares", Presented at the Natl. Conf. on Information \& Communication Technology (NCICT-2011), Bangalore, May 13, 2011.

[3] N. Gnanasankaran, S. Natarajan, K. Alagarsamy, and K. Iyakutti, "Application of COTS components in crystallography," British Journal of Mathematics \& Computer Science, 2012.

[4] N. Gnanasankaran, S. Natarajan, K. Alagarsamy, and K. Iyakutti, "Commercial Off-The-Shelf (COTS) components in software engineering: The software package SCILAB," International Journal of Computer Technology \& Applications, 2013.

[5] D. van der Spoel et al., Gromacs User Manual, version 3.2, 2004. 


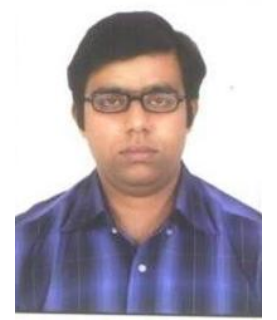

N. Gnanasankaran has a M.C.A. Degree in 2008, from the Computer Centre, Madurai Kamaraj University, Madurai, Tamil Nadu, India. He worked as a Project Assistant at the Bioinformatics Centre, Indian Institute of Science, Bangalore, during the year 2009. Later, he served the M.C.A Department of the New Horizon College of Engineering, Bangalore(2010). Presently, he is an Assistant Professor in the M.C.A. Department of the K.L.N. College of Information Technology, Pottapalayam, Madurai. He has presented several research papers in National and International conferences and has few research publications. He is carrying out research on Commercial Off-The-Shelf (COTS) components in Software Engineering for his doctoral degree.

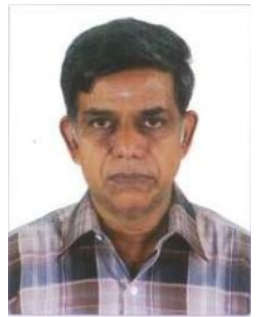

S. Natarajan was born at Muthupettai, Tamilnadu State, India in 1949. He obtained the B.Sc. and M.Sc. degrees from the University of Madras and the Ph.D (1979) (all in Physics) from the Madurai Kamaraj University, India

Joining as a Lecturer in 1976, he rose to the position of Senior Professor and Head and Chairperson of the School of Physics, Madurai Kamaraj University, Madurai and retired in 2010 Presently, he is an Emeritus Scientist (CSIR) in the same Department. He has more than 35 years of experience in Teaching (PG) and Research. His major fields of interest are crystallization of small molecules of biological and non-linear optical materials and the crystallography of small molecules. He has guided several students towards their M.Phil. and Ph.D. degrees. Dr. Natarajan has handled several major research projects funded by UGC, CSIR and DST. He serves as a reviewer for many international journals. He has authored four book chapters. He has published more than 250 research papers in peer-reviewed international journals and has attended scores of National and International Conferences and presented research papers and invited lectures. He has also been a member of the technical/advisory committees of many National Conferences. He has received several awards such as UGC Visiting Associateship and many best paper awards in Conferences. He has visited Norway, Italy, Malaysia and Singapore.

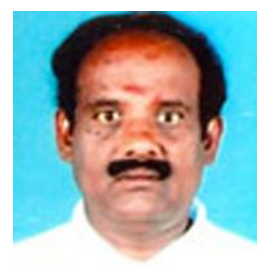

K. Alagarsamy is presently serving as an Associate Professor in the Computer Centre of the Madurai Kamaraj University, Madurai, Tamil Nadu, India. $\mathrm{He}$ received the Ph.D Degree in Computer Applications from the Madurai Kamaraj University, in 2008. His research area is software engineering. He has published 35 papers in International journals. $\mathrm{He}$ is the Chairman of the Board of study in MCA of the Madurai Kamaraj University. Presently, twelve students are working under his guidance for

their doctoral degrees.

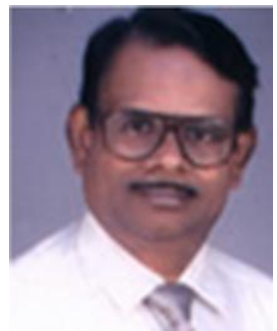

K. Iyakutti received his Ph.D. degree in Physics from the University of Madras, Chennai, Tamil Nadu, India, in 1978. Currently, he is a Professor in the Department of Physics and Nanotechnology of the SRM University, Kattankulathur, Chennai, Tamil Nadu, India. He regularly visits the Institute of Materials Research, Tohoku University, Sendai, Japan.

He joined as a Lecturer in the University of Madras, Department of Nuclear Physics in 1978 and then as Reader in Madurai Kamaraj University in 1984. He was appointed as Professor in 1987 and as Senior Professor in 1998. He has more than 35 years of experience in Teaching (PG) and Research. He has guided many Ph.D., and M.Phil. Scholars. He is a recognized guide in Physics \& Computer Science in the Madurai Kamaraj University. His area of Research is Computational Condensed matter Physics including nanoscience and technology. He has authored more than 200 research papers in International \& National Journals and Conferences. He has visited many research centers abroad.

He was responsible for the initiation of Electronic Band structure Research in South India. He is a Fellow of Tamil Nadu Academy of Sciences. He was an elected member of the syndicate of the Madurai Kamaraj University during 2000-2003. He was the Dean (Academic \& Computerization) at the Madurai Kamaraj University during 2005-08 and NACC Coordinator for Re-accreditation. 